\title{
A semi-phenomenological model to predict the acoustic behavior of fully and partially reticulated polyurethane foams
}

\author{
Olivier Doutres, ${ }^{1, a)}$ Noureddine Atalla, ${ }^{1}$ and Kevin Dong ${ }^{2}$ \\ ${ }_{1}^{1}$ GAUS, Department of mechanical engineering, Université de Sherbrooke, Québec JIK $2 R 1$, Canada \\ ${ }^{2}$ Woodbridge Foam Corporation, Chemical Development Center, 8214 Kipling Avenue, Woodbridge, \\ Ontario L4L 2A4, Canada
}

(Received 30 August 2012; accepted 7 January 2013; published online 1 February 2013)

\begin{abstract}
This paper proposes simple semi-phenomenological models to predict the sound absorption efficiency of highly porous polyurethane foams from microstructure characterization. In a previous paper [J. Appl. Phys. 110, 064901 (2011)], the authors presented a 3-parameter semi-phenomenological model linking the microstructure properties of fully and partially reticulated isotropic polyurethane foams (i.e., strut length $l$, strut thickness $t$, and reticulation rate $R_{w}$ ) to the macroscopic non-acoustic parameters involved in the classical Johnson-Champoux-Allard model (i.e., porosity $\phi$, airflow resistivity $\sigma$, tortuosity $\alpha_{\propto}$, viscous $\Lambda$, and thermal $\Lambda^{\prime}$ characteristic lengths). The model was based on existing scaling laws, validated for fully reticulated polyurethane foams, and improved using both geometrical and empirical approaches to account for the presence of membrane closing the pores. This 3-parameter model is applied to six polyurethane foams in this paper and is found highly sensitive to the microstructure characterization; particularly to strut's dimensions. A simplified micro-/macro model is then presented. It is based on the cell size $C_{s}$ and reticulation rate $R_{w}$ only, assuming that the geometric ratio between strut length $l$ and strut thickness $t$ is known. This simplified model, called the 2-parameter model, considerably simplifies the microstructure characterization procedure. A comparison of the two proposed semi-phenomenological models is presented using six polyurethane foams being either fully or partially reticulated, isotropic or anisotropic. It is shown that the 2-parameter model is less sensitive to measurement uncertainties compared to the original model and allows a better estimation of polyurethane foams sound absorption behavior. (C) 2013 American Institute of Physics. [http://dx.doi.org/10.1063/1.4789595]
\end{abstract}

\section{INTRODUCTION}

Highly porous polyurethane (PU) foams are commonly used as sound absorbers in automotive applications to improve occupant comfort. As shown in Figure 1, a typical PU foam microstructure can be seen as a collection of interlinked struts forming 3D structures as a packing of tetrakaidecahedra cells. ${ }^{1}$ Each cell is connected to others through pores. Materials with $100 \%$ open pores are called "fully reticulated." In this case the interconnectivity between cells is maximal. If some of the pores are closed or partially closed by thin membranes, the material is called "partially reticulated." Cell size and cell interconnectivity (i.e., reticulation rate) are important parameters for sound absorbing efficiency since they will control how the acoustic wave could penetrate inside the porous frame and thus dissipate its energy from visco-thermal couplings.

The wave propagation in rigid-frame porous media is classically modeled under the long-wavelength condition to describe the porous media as an "equivalent fluid." This equivalent fluid is characterized by two macroscopic dynamic properties, the effective density and bulk modulus, which take into account the visco-thermal and inertial couplings between the porous aggregate and the interstitial fluid. The two dynamic properties are based on a set of

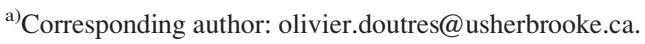

macroscopic physical parameters, known as the non-acoustic parameters. In the case of the classical Johnson-ChampouxAllard (JCA) porous model, ${ }^{2}$ the involved non-acoustic parameters are open porosity $\phi$, static air flow resistivity $\sigma$ $\left[\mathrm{N} . \mathrm{s} / \mathrm{m}^{4}\right]$, static tortuosity $\alpha_{\propto}$, viscous $\Lambda[\mu \mathrm{m}]$, and thermal characteristic lengths $\Lambda^{\prime}[\mu \mathrm{m}]$. These parameters are defined in terms of microstructure geometry (for the porosity and the thermal characteristic length) or microscopic quantities such as fluid particle velocity (for the airflow resistivity, tortuosity, and viscous characteristic length) space-averaged over a representative elementary volume. They are thus intercorrelated and microstructure-dependent. In consequence, they cannot be varied independently to optimize the sound absorption of foams since this will result in non-realistic and non-producible materials. A microstructure based model is needed for such optimization. The motivation of this work is to present a simple model linking two readily measurable microstructure properties of highly porous PU foams to the aforementioned non-acoustic parameters.

Three types of approaches have been proposed in the literature to link microstructure properties of polyurethane foams with non-acoustic parameters and acoustic properties: (i) empirical, ${ }^{3}$ (ii) analytical based on simplified models of the microstructure and wave propagation inside the material (also known as scaling laws), ${ }^{4-6}$ and (iii) numerical homogenization derived in a representative unit-cell. ${ }^{7-11}$ A detailed description of these approaches has been recently given by 


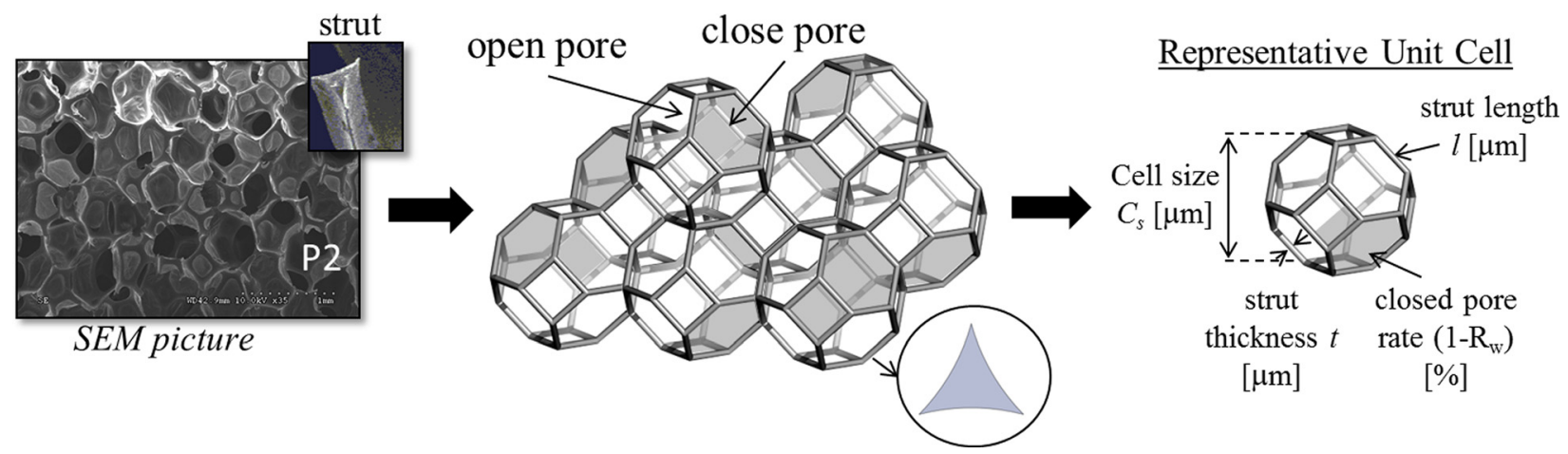

FIG. 1. SEM picture of the partially reticulated polyurethane foam P2 and shape of the idealized unit-cell (PUC).

the authors. ${ }^{12}$ A combination of empirical and analytical methods was also presented in Ref. 12 to link microstructure properties of highly porous isotropic PU foams with nonacoustic macroscopic parameters required in the JCA model. The presented semi-phenomenological (or semi-empirical) model was based on existing scaling laws, validated for fully reticulated PU foams, and improved using both geometrical and empirical approaches to account for the presence of membrane closing the pores. This model, referred to by the 3 -parameter model in this paper, requires measurements of three characteristic microstructure properties of a representative unit-cell as shown in Figure 1; strut's length $l$, strut's thickness $t$, and reticulation rate $R_{w}$ (i.e., open pore content).

Both semi-phenomenological and numerical approaches require the challenging task of characterizing the representative unit-cell of such complex and disordered network that constitutes the porous frame. Direct or inverse approaches can be applied to assess these properties. The direct method is based on measurements made on micrographs: 2D images from optical microscope or scanning electron microscope (SEM) or 3D reconstruction from X-ray tomography techniques (CT-scan). The inverse method is based on measurements of macroscopic non-acoustic properties (e.g., porosity, airflow resistivity...) coupled to calculations from the three aforementioned micro-/macro models; i.e., empirical, analytical, or numerical models. ${ }^{11}$ This latter method provides an effective representative unit-cell that captures the complex microstructure of the porous aggregate being isotropic or anisotropic, fully or partially reticulated. However, this representative unit-cell is not real and its characteristic properties depend on the model used for the inversion. Furthermore, the micro-macro model associated to an inverse characterization of the unit-cell requires macroscopic inputs (i.e., non-acoustic parameters) and thus cannot be used for microstructure based optimization purposes. On the other hand, the direct method has also drawbacks related to the microstructure characterization process: (i) a sophisticated device (e.g., SEM, CT-scan) is required for measuring small microstructure parameters such as strut's thickness and strut's length; (ii) the determination of 3D properties from 2D pictures can lead to systematic errors that can be difficult to quantify; (iii) the closed pore content $\left(1-R_{w}\right)$ is difficult to measure precisely, particularly with CT-scan which hardly detects the thin membranes; (iv) a great number of pictures has to be analyzed to ensure the representativeness of the unit-cell; (v) a large variability in the outputs values can be observed due to possible foam microstructure inhomogeneity; (vi) the microstructure has to be analyzed along the 3 dimensions to estimate the degree of anisotropy (for cell size and closed pore content). Despite all the aforementioned difficulties, the direct method provides a unit-cell that is representative of the real foam microstructure.

The objective of this work is to provide a simple micro-/macro semi-phenomenological model dedicated to highly porous isotropic PU foams being fully or partially reticulated based on simple direct characterization of the unit-cell. This semi-phenomenological approach is preferred in this work since it is more adapted for optimizing large structures as found in the automotive or aeronautic fields. In order to simplify the unit-cell characterization, a 2parameter model for highly porous PU foam is proposed. It is a simplification of the 3-parameter model presented by the author in Ref. 12. It only requires measurements of the cell size $C_{s}$ and reticulation rate $R_{w}$ assuming that the geometric ratio between strut length $l$ and strut thickness $t$ is known. Indeed, considering cell size in place of strut's dimensions considerably simplifies the measurement process and practical use of the model since the cell size parameter is commonly used by chemists. The geometric ratio $B=l / t$, which is related to foam porosity and density, is set constant and is determined from the 15 highly porous PU foams previously investigated in Ref. 12 and having an open porosity ranging from $96.8 \%$ and $99 \%$ and a frame density from $21.5 \mathrm{~kg} \cdot \mathrm{m}^{-3}$ to $29.5 \mathrm{~kg} \cdot \mathrm{m}^{-3}$. To validate both the 3 -parameter and the 2-parameter models, six polyurethane foams, not used during the development of the initial 3-parameter model, are investigated: two are isotropic and fully reticulated; three are isotropic and partially reticulated; and one is anisotropic with possible membranes. Three of these foams have recently been investigated by Perrot et al. and both their microstructure and non-acoustic properties are given in Ref. 11. The other three foams are completely characterized by the authors (using the methods described in Appendix B) and used to investigate the effect of uncertainty in the measured microstructure parameters on the prediction of the sound absorption behavior. The classical Monte Carlo method is used to investigate the impact of microstructure variability on the estimation of the sound absorbing behavior of the foams. It gives an insight on the required precision of the imaging procedure. 


\section{MICROSTRUCTURE CHARACTERIZATION}

Microstructure of the polyurethane foams is analysed from SEM pictures. The SEM technique is preferred in this work because it is more accurate compared to optical method and captures better the thin membranes compared to CTscan. PU foam microstructure is assumed as an idealized packing of tetrakaidecahedra unit-cells (see Figure 1) characterized by $f=14$ faces/cell and $p=5.14$ edges/face and independent of cell density. ${ }^{1,7}$ The PU struts have a triangular concave cross-section shape (i.e., concave Plateau border) characteristic of liquid foams. The void volume at the strut connection is not taken into account; which thus restricts the study to highly porous foams. ${ }^{13}$ However, this assumption leads to simple analytical expressions in the case of the triangular concave cross-section shape.

For a given material, the properties of a representative tetrakaidecahedra unit-cell (also called PUC) are measured from SEM images using the "IMAGE J" software. The PUC properties are the mean cell size $C_{s}[\mu \mathrm{m}]$, the mean strut length $l[\mu \mathrm{m}]$ and thickness $t[\mu \mathrm{m}]$, and the reticulation rate $R_{w}[\%]$. The reader is referred to Ref. 12 for a more complete description of the measurement process. The struts of polyurethane foams having triangular cross-section shape, only the edge $a$ of the triangle is thus measured on SEM pictures. The strut thickness $t$ is the height of this triangle assumed equilateral and is thus given by $t=a \sqrt{3 / 2}$. The reticulation rate $R_{w}$ [\%], which gives the open window content of the material, is estimated by the ratio of the number of open window to the total number of windows visible on the pictures. It is worth mentioning that some PU foams investigated in Ref. 12 and in this paper depict an anisotropy in the reticulation rate: i.e., a different reticulation rate is observed in the longitudinal direction (SEM pictures taken in the plane perpendicular to the wave propagation) and in the transverse direction (SEM pictures taken in the plane parallel to the wave propagation). The reticulation rate considered in this work is the one measured in the longitudinal direction since it characterizes the foam microstructure in the plane impinged by the acoustic wave. The influence of this reticulation anisotropy should be investigated when these foams are subjected to oblique incidence acoustic waves; however this is beyond the scope of this work. The Degree of Anisotropy of the cell (DA) is also estimated for each material. It is defined as the large to small cell radius ratio. ${ }^{12}$ Since we are interested in linking the cell microstructure to the non-acoustic parameters used in simple models (neglecting anisotropy), only polyurethane foams having isotropic cells are considered in the analysis and the used discrimination threshold is set to DA $=1.25$. This limits the analysis to PU foam with a log-normal and sharply peaked distribution of the mean strut length ${ }^{11}$ and thus justifies the use of the idealized isotropic cell shape shown in Fig. 1. Standard deviation and expanded uncertainty of all microstructure properties are estimated from a large number of measurements ${ }^{14}$ carried out on various SEM pictures taken on different positions on the foam surface. Evaluation of the statistics linked to all microstructure parameters is of upmost importance here since it gives an insight on microstructure variability and complexity.
Fifteen materials were studied during the development of the original 3-parameter model: ${ }^{12} 9$ were fully reticulated $\left(R_{w}=100 \%\right)$ and 6 partially reticulated with a reticulation rate ranging from $10 \%$ to $70 \%$. The 15 isotropic PU foams have cell sizes ranging from $500 \mu \mathrm{m}$ to $1600 \mu \mathrm{m}$. These foams are highly porous with an open porosity ranging between $96.8 \%$ and $99 \%$ and a bulk density varying between $21.5 \mathrm{~kg} \cdot \mathrm{m}^{-3}$ and $29.5 \mathrm{~kg} \cdot \mathrm{m}^{-3}$. It was observed that the strut thickness $t$, strut length $l$, and mean pore size increase linearly with cell size for both fully reticulated $\left(R_{w}=100 \%\right)$ and partially reticulated materials $\left(R_{w}<100 \%\right)$. Also, it was found that the strut length to thickness ratio $B=l / t$ and the cell size to window size ratio $C_{s} / W_{m}$ are almost identical for all cell sizes. This indicates that from one cell size to another, the shape of the cells is unchanged and their size parameters are only magnified by a given factor. This observation was expected since the $15 \mathrm{PU}$ foams share similar open porosity and density. Thus, in the case of highly porous PU foams, it is possible to find simple relations between strut dimensions and cell size. From all PU foams considered in this work the strut length $l$ is approximately equal to ${ }^{12}$

$$
l=\frac{C_{s}}{A \sqrt{2}},
$$

with $A=2.33 \pm 0.36$ which is very close (given the great complexity of the microstructure and the measurement process) to the theoretical value of $A=2$ for an idealized tetrakaidecahedra isotropic unit-cell. The strut thickness $t$ is equal to

$$
t=\frac{l}{B}=\frac{C_{s}}{A B \sqrt{2}},
$$

with $B=3.78 \pm 0.53$. These two geometric factors $A$ and $B$ are the basis of the model simplification proposed in this paper. Indeed, the representative unit-cell of highly porous PU foam is now characterized only by the cell size and the reticulation rate; the mean strut thickness and length can be deduced from cell size measurement and Eqs. (1) and (2).

\section{SEMI-PHENOMENOLOGICAL MODELS}

This section recalls the semi-phenomenological expressions related to the 3 -parameter mode ${ }^{12}$ for each non-acoustic parameter involved in the JCA porous model: porosity $\phi$, airflow resistivity $\sigma$, tortuosity $\alpha_{\propto}$, viscous $\Lambda$, and thermal $\Lambda^{\prime}$ characteristic lengths. For completeness, the JCA porous model is briefly recalled in Appendix A. The micro-/macro expressions are also written in terms of the two geometric factors $\mathrm{A}$ and $\mathrm{B}$ and the cell size to constitute the simplified 2-parameter model.

\section{A. Porosity $\phi$}

The porosity $\phi$ is defined as the ratio of the fluid volume $V_{f}$ to the total volume $V_{t}$. In the case of highly porous $\mathrm{PU}$ foams, the unit-cell is assumed to have a tetrakaidecahedra unit-cell shape and the porosity is given by 


$$
\phi=\frac{V_{f}}{V_{t}}=1-\frac{\rho_{1}}{\rho_{s}}=1-\rho_{r}=1-C^{\rho}\left(\frac{t}{l}\right)^{2}
$$

with $\rho_{l}$ the bulk density of the foam, $\rho_{s}$ the density of the strut material, and $\rho_{r}$ the relative density which can be expressed in terms of strut thickness $t$ and strut length $l$ forming the cells. This expression can thus simply be written in terms of the geometric factor $B$ as

$$
\phi=1-\frac{C^{\rho}}{B^{2}} .
$$

$C^{\rho}$ is a constant which depends on the microscopic mechanical properties of the foam, such as cell shape, strut cross section and joint region shape. As observed on SEM images and shown in Fig. 1, the struts of PU foams have a triangular concave cross-section shape and the constant $C^{\rho}$ is chosen equal to $C^{\rho}=C_{t}{ }^{\rho}=(2 \sqrt{3}-\pi) / \sqrt{2}{ }^{12}$ Porosity of these highly porous open-cell foams does not depend on the cell size nor on reticulation rate but only on the cell shape and geometric ratio between strut thickness and length. Indeed, the effect of membranes is not taken into account here since the membrane volume can be neglected in the calculation of the frame volume $V_{s}$. Equations (3) and (4) are in consequence also used for partially reticulated polyurethane foams. Figures 2(a) and 2(b) show that the geometric factor $B$ is found relatively constant for the $15 \mathrm{PU}$ foams (M1 to M15 see Ref. 12) for which the density is ranging between $21.5 \mathrm{~kg} \cdot \mathrm{m}^{-3}$ and $29.5 \mathrm{~kg} \cdot \mathrm{m}^{-3}$ and the porosity between $96.8 \%$ and $99 \%$. As already mentioned in Ref. 12, Figure 2(b) confirms that modeling the struts of these $15 \mathrm{PU}$ foams with triangular-concave cross-section shape gives a good estimate of the porosity. It also shown that Eq. (4) deviates slightly from measurements for $\phi<98 \%$ and confirms that the model, which does not account for the void volume at the strut connection, is more adapted to highly porous foams. Comments about the new PU foams P1 to P3 (white points) and R1 to R3 (grey squares) are given in Sec. V.

\section{B. Thermal length $\Lambda^{\prime}$}

As for the porosity, the thermal characteristic length $\Lambda^{\prime}$ (also referred to as the hydraulic radius) can be determined from simple geometrical calculations since it is defined as twice the average ratio of the cell volume $V_{f}$ to their wet surface $A_{t}{ }^{2}$ It is thus possible to account for the effect of the thin membranes closing the cell pores on the wet surface $A_{t}$ such as

$$
\Lambda^{\prime}=\frac{2 V_{f}}{A_{t}}=\frac{2 V_{f}}{A_{s}+\left(1-R_{w}\right) A_{w}}
$$

with $A_{s}$ the surface of the struts and $A_{w}$ the total surface of the pores weighted here by the closed pore content $\left(1-R_{w}\right)$. $A_{s}$ can be calculated from the perimeter of the strut $P_{s}$ for a given cross-section shape. Assuming a tetrakaidecahedra unit-cell, the surface of the 36 struts per cell $A_{s}$, is given by $A_{s}=36 l P_{s} / 3$; the " $1 / 3$ " coefficient accounts for the fact that one strut is shared between three cells. For struts having a triangular concave cross-section shape $P_{s}$ is equal to $4 \pi t / \sqrt{3}$. Note that the strut thickness $t$ is the height of the equilateral triangle with edge $a$ and concavity radius $R=a$. The total surface of the pores is determined from the total surface of the cell $A_{c}$ as $A_{w}=A_{c}-A_{s}$ with $A_{c}=(6+12 \sqrt{3}) l^{2}$. It is worth noting that Eq. (5) accounts for the presence of

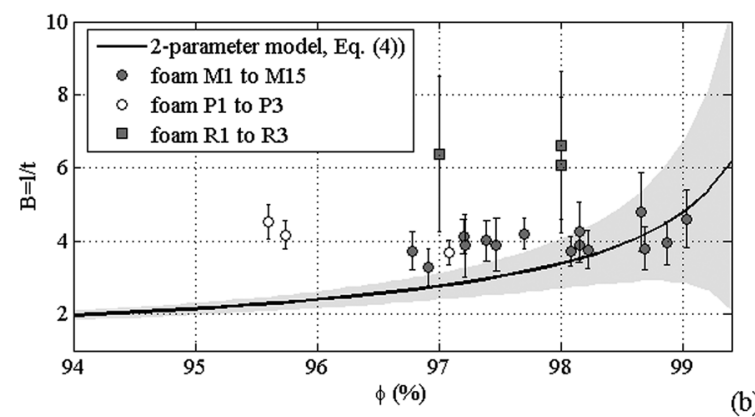

(b)

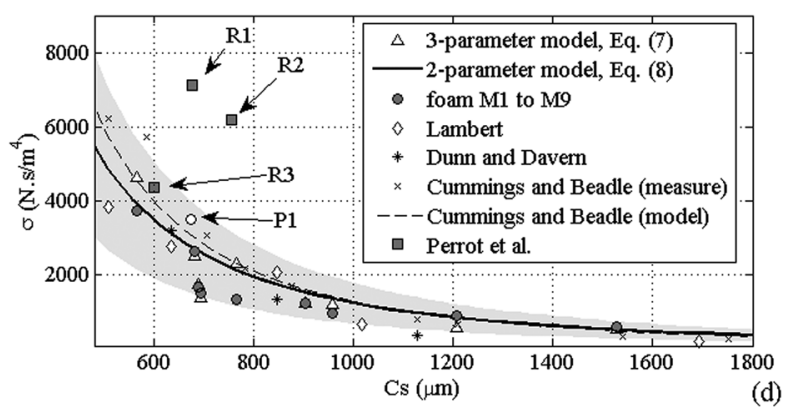

FIG. 2. (a) Measured geometric coefficient $B$ versus frame bulk density; (b) Measured geometric coefficient $B$ versus porosity. The model considers a PUC with triangular concave struts (Eq. (4) with $C^{\rho}=C_{t}^{\rho}=(2 \sqrt{3}-\pi) / \sqrt{2}$ ); (c) Thermal characteristic length versus cell size for fully reticulated polyurethane foams $\left(R_{w}=100 \%\right)$. The 3-parameter model involves measurements of $l, t$ and $R_{w}$; the 2-parameter model involves measurements of $C_{s}$ and $R_{w}$; (d) Airflow resistivity versus cell size for fully reticulated polyurethane foams $\left(R_{w}=100 \%\right)$; (grey area) propagation of microstructure measurements uncertainties. White circles and grey squares: measurements of foams P1, R1, R2, and R3 presented in Sec. IV (note that foams R1 and R2 are partially reticulated). 
membranes from geometrical considerations only and does not involve empirical coefficients.

Using Eqs. (1) and (2), Eq. (5) can be written in terms of cell size $C_{s}$, reticulation rate $R_{w}$ and two geometric ratios $(A$ and $B)$ as

$$
\begin{aligned}
\Lambda^{\prime} & =C_{s} \frac{C_{1}}{C_{2}-R_{w} C_{3}} \\
& =C_{s} \frac{8\left(1-(2 \sqrt{3}-\pi) / B^{2} \sqrt{2}\right) / 3 A}{(1+2 \sqrt{3})-R_{w}(1+2 \sqrt{3}-4 \pi / B \sqrt{3})} .
\end{aligned}
$$

For fully reticulated PU foams, the thermal length to cell size ratio $\Lambda^{\prime} / C_{s}=C_{1} /\left(C_{2}-C_{3}\right)$ is equal to 0.59 which is very close to the 0.56 found experimentally in Ref. 12 (see Sec. II.B.1 in Ref. 12). The good correlation between Eq. (6) and measurements for the 9 fully reticulated foams M1 to M9 is confirmed in Figure 2(c). It is also observed that the uncertainty related to Eq. (6) increases with the foam cell size (see grey area). Note that for poorly resistive foams having large cell size $\left(C_{s}>1.2 \mathrm{~mm}\right)$, the estimation of $\Lambda^{\prime}$ from acoustic indirect characterization technique (see Appendix B) is more difficult and its associated measurement uncertainty is increased. This mainly explains the differences observed between measurements and simulations for cell size superior to $1 \mathrm{~mm}$.

\section{Airflow resistivity $\sigma$}

The scaling law proposed by Lind-Nordgren and Göransson ${ }^{5,6}$ to link microstructure properties with the nonacoustic macroscopic airflow resistivity parameter is used for fully reticulated foams and improved empirically to account for the presence of thin membranes closing the cell windows ${ }^{12}$

$$
\sigma=\sigma^{\prime}\left(\frac{1}{R_{w}}\right)^{1.1166}=C^{\beta}\left(C_{r}^{\rho} \frac{t}{l^{2}}\right)^{2}\left(\frac{1}{R_{w}}\right)^{1.1166} .
$$

The original scaling law, i.e., $\sigma^{\prime}=\mathrm{f}(t, l)$, combines different simplified models of wave propagation inside the microstructure and has a large number of implicit assumptions: ${ }^{12}$ propagation in a cylindrical pore, material with low tortuosity $\left(\alpha_{\propto} \approx 1\right)$, high porosity $(\phi \approx 100 \%)$, and wave propagation perpendicular to strut with a circular cross-section shape. It is thus adapted to fully reticulated materials. Note that in Eq. (7), $C_{r}{ }^{\rho}$ is associated to strut with circular crosssection shape $\left(C_{r}{ }^{\rho}=3 \pi / 8 \sqrt{2}\right)$ because it is based on analytical calculations derived for fibers. Furthermore $C^{\beta}$ in Eq. (7) is equal to $128 \alpha_{\propto} \eta / c_{g}^{2}$ (note that $C^{\sigma}$ in Ref. 12 is changed for $C^{\beta}$ here to avoid any confusions with the airflow resistivity notation) with $\alpha_{\propto}=c_{g}=1$. Still, Eq. (7) gives surprisingly a satisfactory prediction of the airflow resistivity for the nine studied fully reticulated polyurethane foams as shown in Figure 2(d) (compare grey circles with white triangles).

Using Eqs. (1) and (2), Eq. (7) can be written in terms of cell size $C_{s}$, reticulation rate $R_{w}$ and two geometric ratios $(A$ and $B$ ) as

$$
\sigma=C^{\beta}\left(C_{r}^{\rho} \frac{A \sqrt{2}}{B}\right)^{2}\left(\frac{1}{C_{s}}\right)^{2}\left(\frac{1}{R_{w}}\right)^{1.1166} .
$$

One can appreciate in Figure 2(d), that Eq. (8) associated to the cell size measurement and the two geometric factors A and B (i.e., 2-parameter model) provide a fairly good estimate of the airflow resistivity of the 9 fully reticulated polyurethane foams measured by the authors (i.e., foam M1 to M9 (Ref. 12)). This expression is also validated compared to the measurements of highly porous fully reticulated PU foams provided by Cummings and Beadle, ${ }^{3}$ Lambert, ${ }^{4}$ and Dunn and Davern. ${ }^{15}$ Note that in Fig. 2(d), the 2-parameter expression (Eq. (8)) is derived for $R_{w}=100 \%$. The airflow resistivity of foams $\mathrm{R} 1$ and $\mathrm{R} 2$ is not correctly estimated since these foams are partially reticulated $\left(R_{w}<100 \%\right)$ as it will be discussed in Sec. V. The grey area represents the uncertainty on the determination of $\sigma$ due to uncertainties on the parameters $C_{s}, A$, and $B$. It thus accounts for the variability in cell size but also in density and porosity. In addition, the inverse proportionality of the airflow resistivity with the square of the cell size, described in the scaling law of Eq. (8), agrees with both the empirical law provided by Cummings and Beadle (note that the pore size $a$ in Ref. 3 corresponds to half the cell size in this paper: $a=C_{s} / 2$ ) (see Eq. (17.a) in Ref. 3) for polyurethane foams and the experimental observations carried out by Bonnet et al. ${ }^{16}$ on aluminum foams. This inverse proportionality also explains that the uncertainty related to the estimation of $\sigma$ increases with decreasing cell size. It is worth noting that the scaling law of Eq. (8), applied to fully reticulated foams, gives similar results compared to the empirical expression given by Cummings and Beadle as shown in Fig. 2(d) (compare dashed and dash-dotted curves). The large uncertainty on the airflow resistivity estimation observed for PU foams having small cell size inferior to $700 \mu \mathrm{m}$ is in good agreement with the large scattering in airflow resistivity measurement observed in Figure 2(d) (compare foams with similar cell size measured by Doutres et al., ${ }^{12}$ Lambert, ${ }^{4}$ and Cummings and Beadle $^{3}$ ). Note that in this work, only PU foams are investigated but Eqs. (4), (6), and (8) can also be used for other highly porous foams with identical cell shape (e.g., aluminum foams) as long as the right geometric factors $A$ and $B$ are used.

\section{Viscous length $\Lambda$ and tortuosity $\alpha_{\propto}$}

Both viscous characteristic length and tortuosity depend on the local fluid velocity in the porous aggregate. Since the complex microstructure of real PU foam prevents accurate analytical representation of the velocity field, an empirical approach is chosen in Ref. 12 to estimate these two properties.

The tortuosity has been found constant $\left(\alpha_{\propto} \approx 1.05\right)$ for highly porous and fully reticulated PU foams. However it is shown to increase with the close pore content $\left(1-R_{w}\right)$ and is given empirically as

$$
\alpha_{\infty}=1.05\left(\frac{1}{R_{w}}\right)^{0.3802}
$$

As for the tortuosity, the ratio between the thermal and the viscous characteristic lengths of fully reticulated PU foams is found constant $\left(n=\Lambda^{\prime} / \Lambda \approx 1.55\right){ }^{12}$ Thus, the 
viscous length of fully reticulated PU foams can be derived from the geometrical estimation of the thermal length of Eq. (5) divided by $n=1.55$. Presence of membranes closing the pores decreases the viscous characteristic length and increases the characteristic length ratio $n\left(n=\Lambda^{\prime} / \Lambda\right) .{ }^{12}$ The constant relation between the two characteristic lengths found in the case of fully reticulated materials, i.e., $n=1.55$, can no longer be used for partially reticulated materials. From the 6 partially reticulated materials used in Ref. 12, an empirical expression of the ratio $n$ is derived in terms of the closed pore content. It is given by

$$
n=\frac{\Lambda^{\prime}}{\Lambda}=1.55\left(\frac{1}{R_{w}}\right)^{0.6763} .
$$

The viscous characteristic length $\Lambda$ for both fully and partially reticulated PU foams is obtained from Eqs. (5) and (10) or from Eqs. (6) and (10) if the simplified 2-parameter model is used.

\section{TEST MATERIALS}

In order to validate the two simple micro-/macro semiphenomenological models, six polyurethane foams (named P1-P3 and R1-R3) which have not been used during the development of the first 3-parameter model are investigated.

As for foams M1 to M15, the three PU foams P1, P2, and $\mathrm{P} 3$ are also provided by the Woodbridge Group. These foams have a bulk density in the range of the PU foams used in the first characterization set (see Table I). However, the porosity of foams P2 and P3 is around 95\% and slightly below the minimum value of the first PU set. The microstructure properties of these foams are measured from SEM pictures as described in Sec. II and are given in Table I. Strut's length and thickness are also measured in order to apply the 3-parameter model and compare it with the simplified 2-parameter model presented in this paper. Foam P1 is a fully reticulated PU foam with a small cell size $\left(C_{s}=673 \mu \mathrm{m}\right)$. Foam P2 is partially reticulated foam with very small cell size $\left(C_{s}=616 \mu \mathrm{m}\right)$ and a low reticulation rate $\left(R_{w}=32 \%\right)$. Foam P3 is partially reticulated foam with very large cell size $\left(C_{s}=1710 \mu \mathrm{m}\right)$ and a very low reticulation rate $\left(R_{w}=5 \%\right)$. It is worth mentioning that these three foams are at the limits of the range of microstructure properties used in the first characterization set (i.e., for materials M1 to $\mathrm{M} 15,500 \mu \mathrm{m}<C_{s}<1600 \mu \mathrm{m}$ and $10 \%<R_{w}<100 \%$ ). Foams P1, P2, and P3 are considered isotropic with a $\mathrm{DA}<1.25$.
The PU foams R1, R2, and R3 are taken from Ref. 11. They are used in this work because they are highly porous PU foams with tetrakaidecahedra unit-cell shape and both their bulk density and porosity fall in the range of the first characterization set (i.e., foams M1 to M15). Note that their microstructure and non-acoustic properties have been fully characterized in Ref. 11. Furthermore, it allows confronting the proposed semi-phenomenological relations with the hybrid numerical model presented in Ref. 11 and testing their applicability to other fully and partially reticulated foams being isotropic and even anisotropic. The cell size $C_{s}$ and strut length $l$ of these foams have been measured in Ref. 11 from photomicrograph taken with an optical microscope. This may explain the large associated uncertainties compared to our SEM measurements (in Table I, compare, for example, the measurement uncertainties associated for foams P1 and R1). However, the strut thickness $t$ of these foams is also estimated from SEM because of lateral borders issues associated to the use of an optical microscope. Values of $l$ and $t$ reported in Table I are called $L_{m}$ and $2 r_{m}$ in Ref. 11. The cell size is deduced from $L_{m}$ as $C_{s}=(A \sqrt{2}) L_{m}$. According to Perrot et al., foam R1 is partially reticulated with isotropic cells, foam R2 is highly anisotropic $(\mathrm{DA}=1.75)$ and foam $\mathrm{R} 3$ is isotropic with residual membranes. The reticulation rates $R_{w}$ are not measured in Ref. 11 and thus are considered equal to $100 \%$ as a first guess. It is worth noting that the two foams R1 and R2 seem unsuitable for the two semi-phenomenological models since (i) the reticulation rate of these foams has not been measured and (ii) the foam R2 is not isotropic. Nonetheless, the proposed semi-empirical models are applied to these two foams to test their limits of applicability.

In summary, the studied six foams can be merged into three different categories: foams P1 and R3 are isotropic and fully reticulated foams with possible residual membranes; foams P2, P3, and R1 are isotropic and partially reticulated; foam R2 is anisotropic (no information is given in Ref. 11 about its reticulation rate). It is worth noting that most of these foams has very small cell size (i.e., $C_{s}<800 \mu \mathrm{m}$ ).

The non-acoustic properties of foams P1-P3 have been measured according to the procedure given in Ref. 12 and recalled in Appendix B. The non-acoustic properties of foams R1-R3 are given in Ref. 11. They have been measured using direct techniques for porosity and airflow resistivity and indirect techniques (inversion of the JCAL model) for the tortuosity and the two characteristic viscous and thermal lengths.

TABLE I. Non-acoustic and microstructure properties of six PU foams (properties of foams R1, R2, and R3 are taken from Ref. 11). Measurements are performed from SEM for foams P1-P3 and from optical microscope for foams R1-R3 (except $t$ determined from SEM).

\begin{tabular}{|c|c|c|c|c|c|c|}
\hline Foam & P1 & $\mathrm{P} 2$ & P3 & $\mathrm{R} 1$ & $\mathrm{R} 2$ & R3 \\
\hline Bulk density $\rho_{1}\left(\mathrm{~kg} \cdot \mathrm{m}^{-3}\right)$ & $28.9( \pm 0.2)$ & $28.9( \pm 0.2)$ & $30.5( \pm 0.2)$ & $\sim 28$ & $\sim 28$ & $\sim 28$ \\
\hline Porosity $\phi(\%)$ & $95.6( \pm 1)$ & $95.7( \pm 1)$ & $97.1( \pm 1)$ & $98( \pm 1)$ & $97( \pm 1)$ & $98( \pm 1)$ \\
\hline Cell size $C_{s}(\mu \mathrm{m})$ & $673( \pm 35)$ & $616( \pm 28)$ & $1710( \pm 161)$ & $676( \pm 138)$ & $755( \pm 188)$ & $600( \pm 138)$ \\
\hline Degree of anisotropy DA & $1.12( \pm 0.06)$ & $1.14( \pm 0.07)$ & $1.25( \pm 0.18)$ & - & $1.75( \pm-)$ & - \\
\hline Strut length $l(\mu \mathrm{m})$ & $208( \pm 16)$ & $209( \pm 13)$ & $550( \pm 37)$ & $205( \pm 42)$ & $229( \pm 57)$ & $182( \pm 42)$ \\
\hline Strut thickness $t(\mu \mathrm{m})$ & $46( \pm 4)$ & $50( \pm 4)$ & $149( \pm 8)$ & $31( \pm 7)$ & $36( \pm 8)$ & $30( \pm 6)$ \\
\hline Reticulation rate $R_{w}(\%)$ & $100(-)$ & $32( \pm 4)$ & $5( \pm 2)$ & - & - & $\sim 100$ \\
\hline
\end{tabular}


Figure 3 presents simulations of the sound absorption coefficient of the six foams derived from the JCA model (see Appendix A) using the measured non-acoustic properties. The simulations are compared to measurements performed in an impedance tube according to standard ISO-10534-2. ${ }^{17}$ All samples are 1 inch-thick. The grey areas represent the propagation of the uncertainties on the non-acoustic parameter measurements to the simulated absorption curves. The good correlation between measurements and simulations for foams P1 to P3 shown in Figs. 3(a)-3(c) indicates that the measured non-acoustic properties can be considered as reference. Figs. 3(d)-3(f) present the JCA simulations associated to the measured non-acoustic properties for foams R1 to R3. Because the sound absorption coefficient measurements of materials R1 to R3 are not available, the simulations presented in Figs. 3(d)-3(f) will be used to evaluate the accuracy of sound absorption predictions using both the 3-parameter and 2-parameter models.

\section{RESULTS AND DISCUSSION}

All non-acoustic parameters of the six PU foams are estimated from the microstructure properties using the two micro-/macro semi-phenomenological models and are compared to measurements. Figure 4 regroups these non-acoustic properties for the six PU foams. The non-acoustic parameters estimated from the 3-parameter or 2-parameter models are shown with circles and triangles, respectively. The efficiency of the two models is first analyzed in terms of the 4 isotropic foams with known reticulation rate: $\mathrm{P} 1, \mathrm{P} 2, \mathrm{P} 3$, and $\mathrm{R} 3$. The results on the two other foams R1 and R2 will be discussed at the end of this section.
Fig. 4 shows that, for the four isotropic PU foams with known reticulation rate $R_{w}$ (i.e., P1-P3 and R3), the 3 -parameter model gives a relatively good estimate for the porosity, the tortuosity (except for foam P3) and the two characteristic lengths. The good estimation of the thermal characteristic length for both fully and partially reticulated foams validates the geometrical approach to account for the presence of membranes closing the pores cell (an empirical approach is not used for this parameter as mentioned previously). The overestimation of the tortuosity for foam P3 can be due to the fact that its reticulation rate is very low $\left(R_{w}=5 \%\right)$ and out of the range of the first characterization set used to derive the empirical expression of Eq. (9) (i.e., for materials M10 to M15, $10 \%<R_{w}<100 \%^{12}$ ). Fig. 4(b) indicates that the airflow resistivity is underestimated for all materials. These discrepancies can be attributed to the great simplicity of Eq. (7) (i.e., idealized PUC shape, strong assumptions associated to the analytical models,...) and to the direct method chosen to determine the characteristic microstructure properties of the representative unit cell (i.e., $C_{s}, l$ and $t$ ). Indeed, in the case of fully reticulated PU foams for example, the uncertainty on the estimation of the airflow resistivity can be large for small cell size (see Figure 2(d)) and results mainly from (i) the measurement uncertainties on strut length $l$ and thickness $t$ and (ii) the statistical variations of $l$ and $t$ within the porous aggregate reflecting the complexity of the foaming process. Fig. 5 clearly shows the consequence of an airflow resistivity underestimation on the simulated sound absorption for the fully reticulated foams P1 and R3 and for the partially reticulated foam P2; the sound absorption coefficient is shown to be underestimated for frequencies below the first sound absorption pick where the
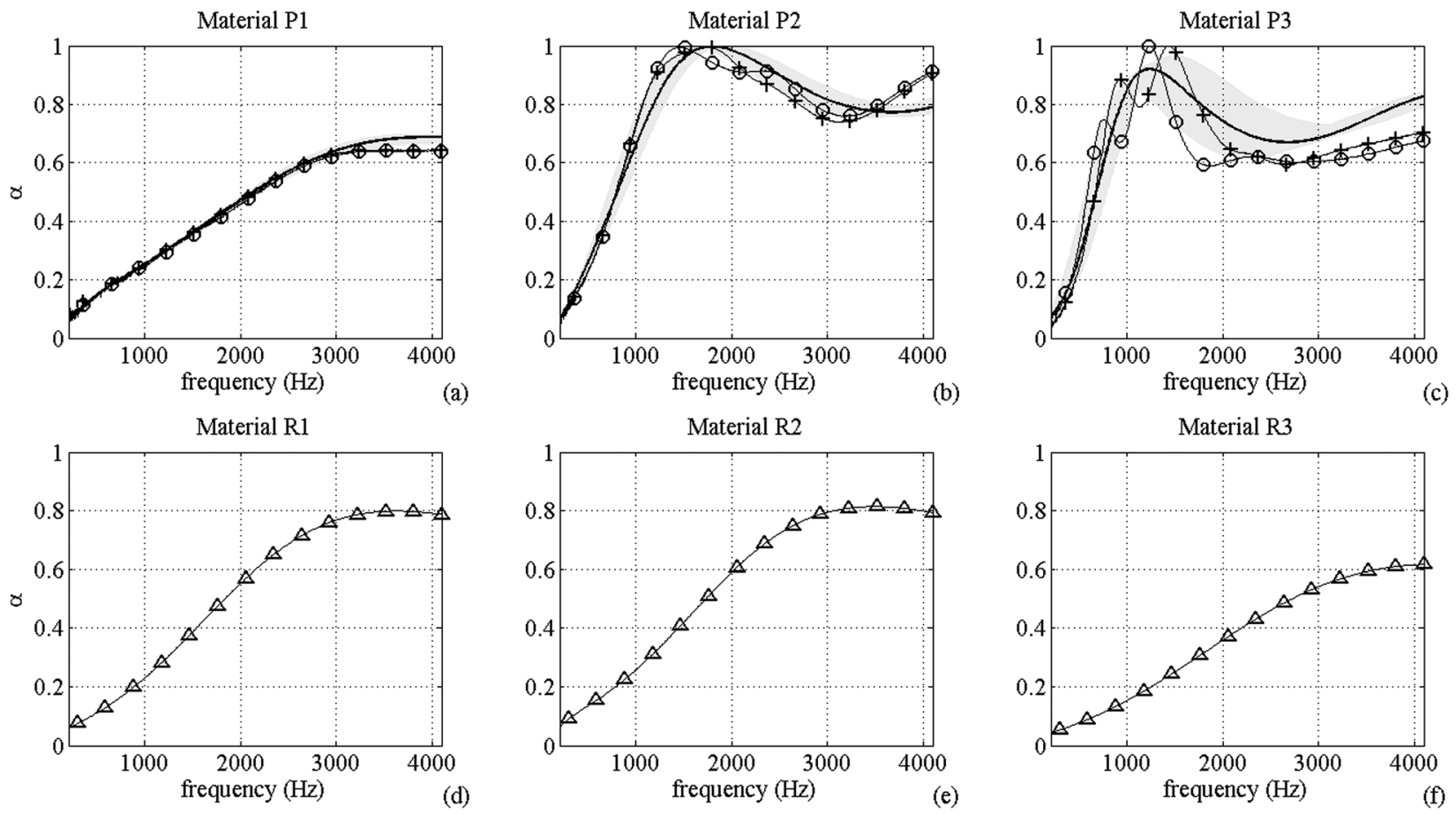

FIG. 3. Sound absorption coefficient of 1 in. thick PU foam layer. Simulations are based on the measured non-acoustic properties: (thick continuous line for (a)-(c) and $\Delta$ for (d)-(f)) JCA model; (grey area) propagation of non-acoustic measurement uncertainties; $(\mathrm{O},+)$ impedance tube measurements (test of two different samples). 

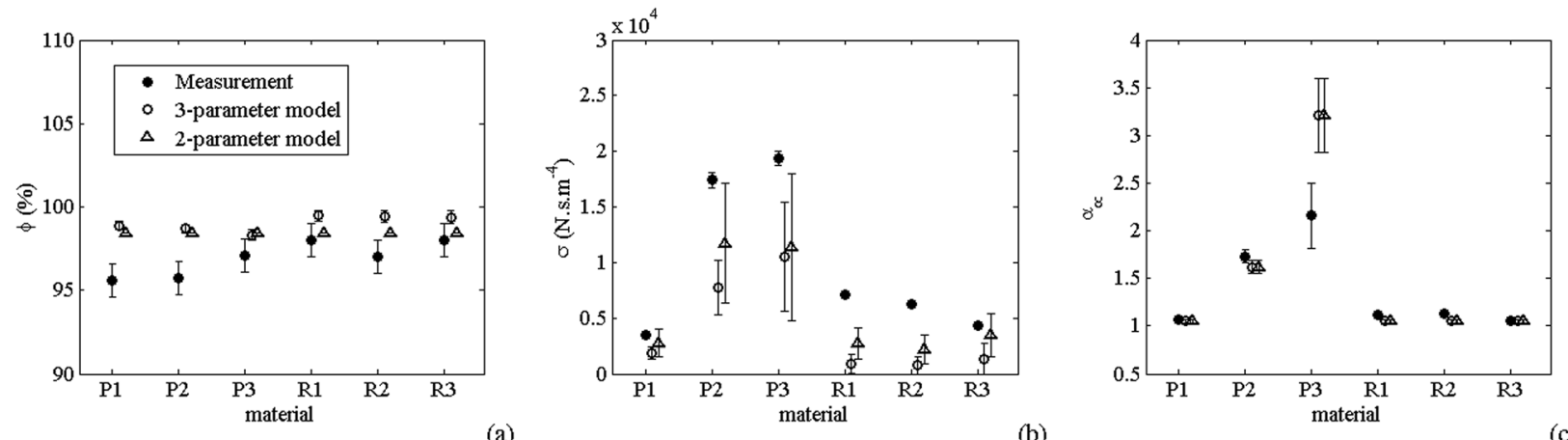

(a)

(b)
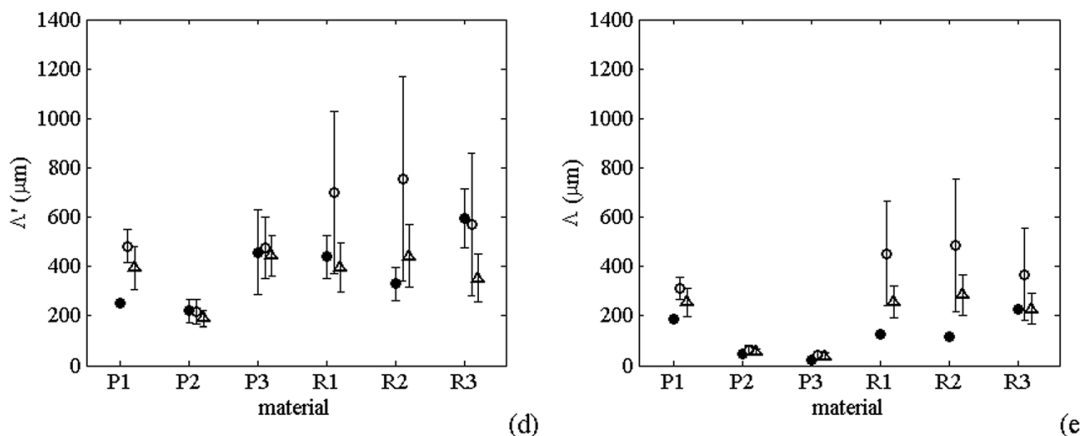

(e)

FIG. 4. Six PU foams P1-P3 and R1-R $3{ }^{11}$ measured non-acoustic properties and estimation from the proposed micro-/macro models; 3-parameter and 2-parameter. The reported uncertainties are based on a standard uncertainty multiplied by a coverage factor $k=2$, providing a level of confidence of approximately $95 \%$.

airflow resistivity influence is predominant. The influence of the airflow resistivity underestimation is less visible in the case of the partially reticulated foam $\mathrm{P} 3$ as shown in Fig. 5(c). This tends to demonstrate that, for these poorly reticulated foam $\left(R_{w}=5 \%\right)$, the large amount of constrictions in the foams microstructure gives more weight to the viscous characteristic length $\Lambda$ for describing the viscous losses in the porous aggregate; characteristic length
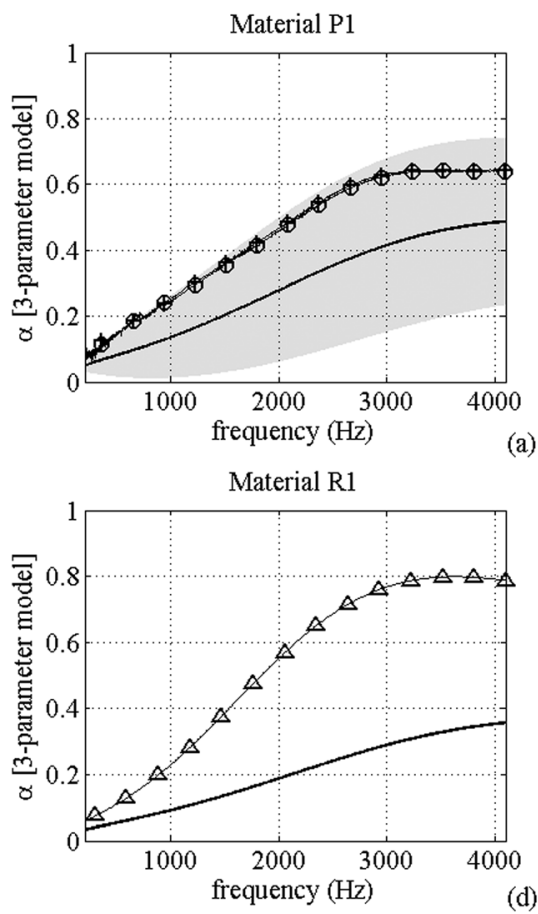

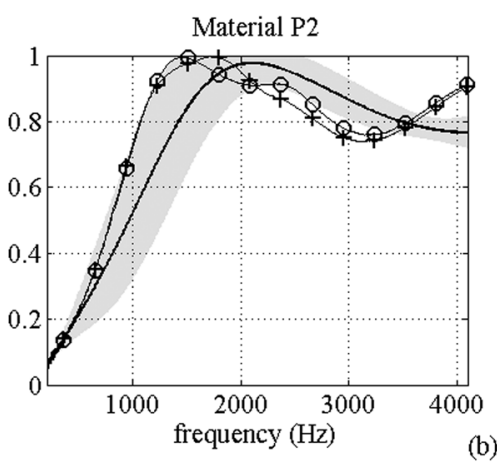

(b)
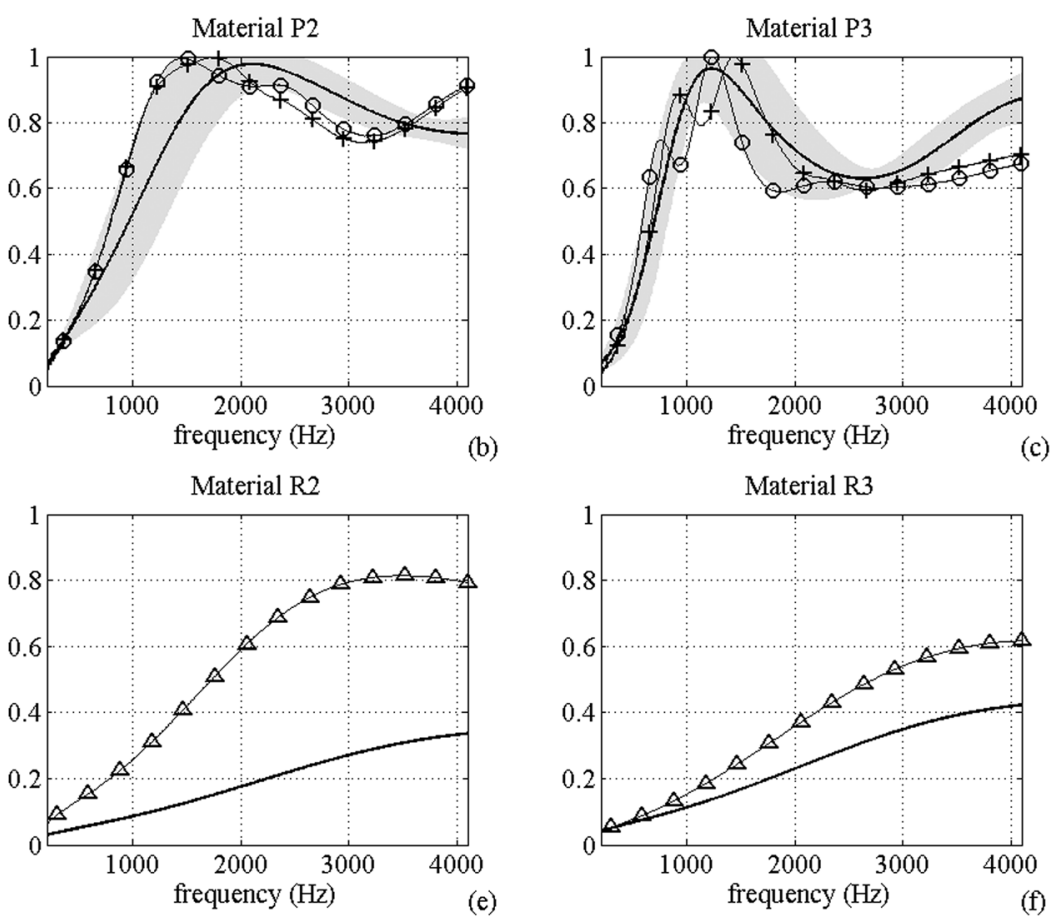

FIG. 5. Sound absorption coefficient of 1 in. thick PU foam layer. Simulations are based on the non-acoustic properties determined from the 3-parameter model: (thick continuous line) JCA model; (grey area) propagation of microstructure measurements uncertainties; $(\mathrm{O},+)$ impedance tube measurements; $\triangle \mathrm{JCA}$ simulation associated to the measured non-acoustic parameters. 
which is accurately estimated by the 3-parameter model as shown in Fig. 4(e). The impact of the microstructure variability is also presented in Figs. 5(a)-5(c) (see grey surface). It is estimated from the Monte-Carlo method associated to $\mathrm{M}=1 \mathrm{e}^{5}$ numerical trials and normal probability density function of the input microstructure quantities. ${ }^{14}$ The sensitivity analysis is not performed on foams R1-R3 since no sufficient microstructure data are available in Ref. 11 to construct the input probability density functions. Figs. 5(a)5(c) show that the influence of the standard deviation on microstructure properties is more pronounced for the fully reticulated foam.

The 2-parameter model provides similar non-acoustic properties compared to the 3-parameter model as shown in Fig. 4. However, the airflow resistivity is better estimated (see Fig. 4(b)) which allows a better prediction of the sound absorption behavior for the two fully reticulated foams P1 and R3 and the partially reticulated foam $\mathrm{P} 2$ as presented in Figs. 6(a), 6(b), and 6(f). The estimation of the sound absorption of foam P3 is not clearly improved since it has been shown previously that the viscous length governs the viscous dissipation mechanisms. Comparison between the two material sets P1-P3 and R1-R3 allows one to better appreciate the limitations of the 3-parameter model compared to the 2-parameter model. Indeed, the 3-parameter model requires strut thickness measurement which can be difficult to assess even with an SEM device. In the case of polyurethane foams, for example, struts have a triangular concave cross-section shape which height may vary along the strut length. ${ }^{18}$ Furthermore, sides of the strut are measured from $2 \mathrm{D}$ images of $3 \mathrm{D}$ structures which can result in a bias in the strut thickness estimation. For example, Table I shows that foams P1 and R1 which share similar bulk density, cell size and strut length, still have a noticeable discrepancy in strut thickness. The smaller strut thickness of foam R1 measured by Perrot et al. from SEM micrographs ${ }^{11}$ could be attributed to the fact that they ignored a light zone situated at the peripheral of the struts that was attributed to membranes (see appendix C in Ref. 11). The strut's dimension measurements of foams R1-R3 lead to a strut length to thickness ratio equal approximately to $B=6.34 \pm 1.99$ for the three foams (see Fig. 2(b)). This coefficient seems overestimated when compared to the one found around $B=3.78 \pm 0.53$ for the PU foams of the first characterization set (i.e., M1-M15 (Ref. 12)) and to the one found around $B=4.11 \pm 0.41$ for foams P1-P3. This apparent overestimation of the ratio $B$ (i.e., underestimation of the mean strut thickness) is thus responsible for the large underestimation of the airflow resistivity of foam R3 derived with the 3-parameter model (see Fig. 4(b)). Indeed, using the proposed geometric ratio $B=3.78$ and the 2-parameter model provides a good estimation of the airflow resistivity for foam R3 as shown in Fig. 2(d) (compare solid line with grey square) and Fig. 4(b) and a good estimation of its sound absorption (see Fig. 6(f)). This suggests that the measurement technique to assess the strut thickness may have an important influence on the 3-parameter model outputs. Because of the difficulty to estimate the strut thickness from micrographs, it is worth mentioning that for highly porous PU foams, the scale factor $B$ required in the 2-parameter model can be estimated from porosity measurement by inverting equation (4). In the case of foams R1-R3, this
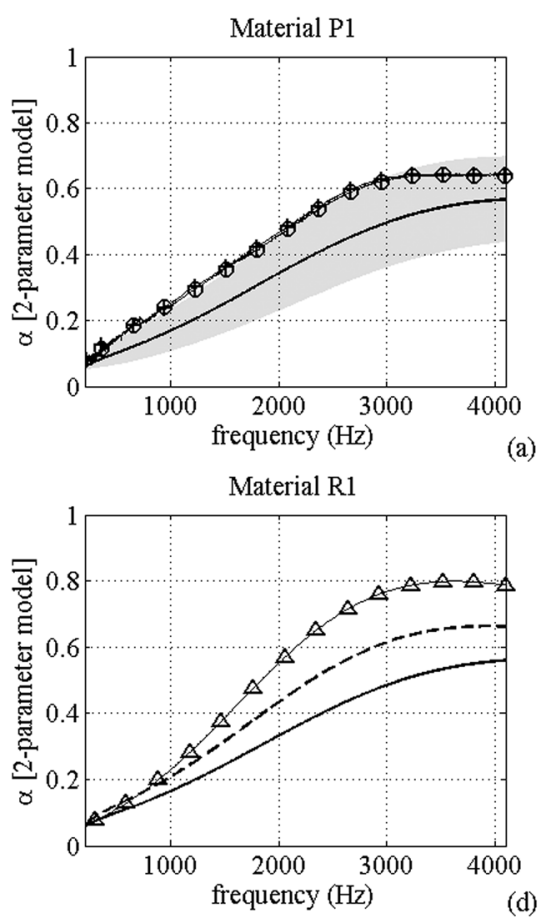

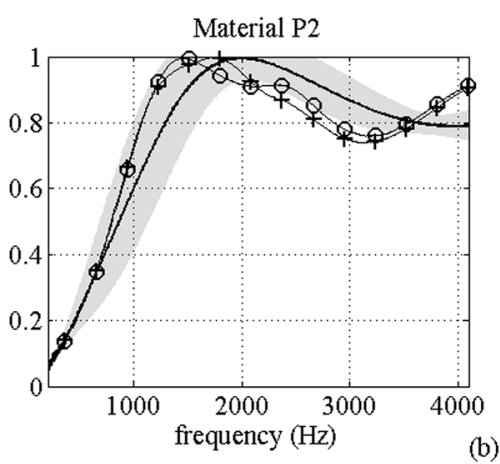

(b)
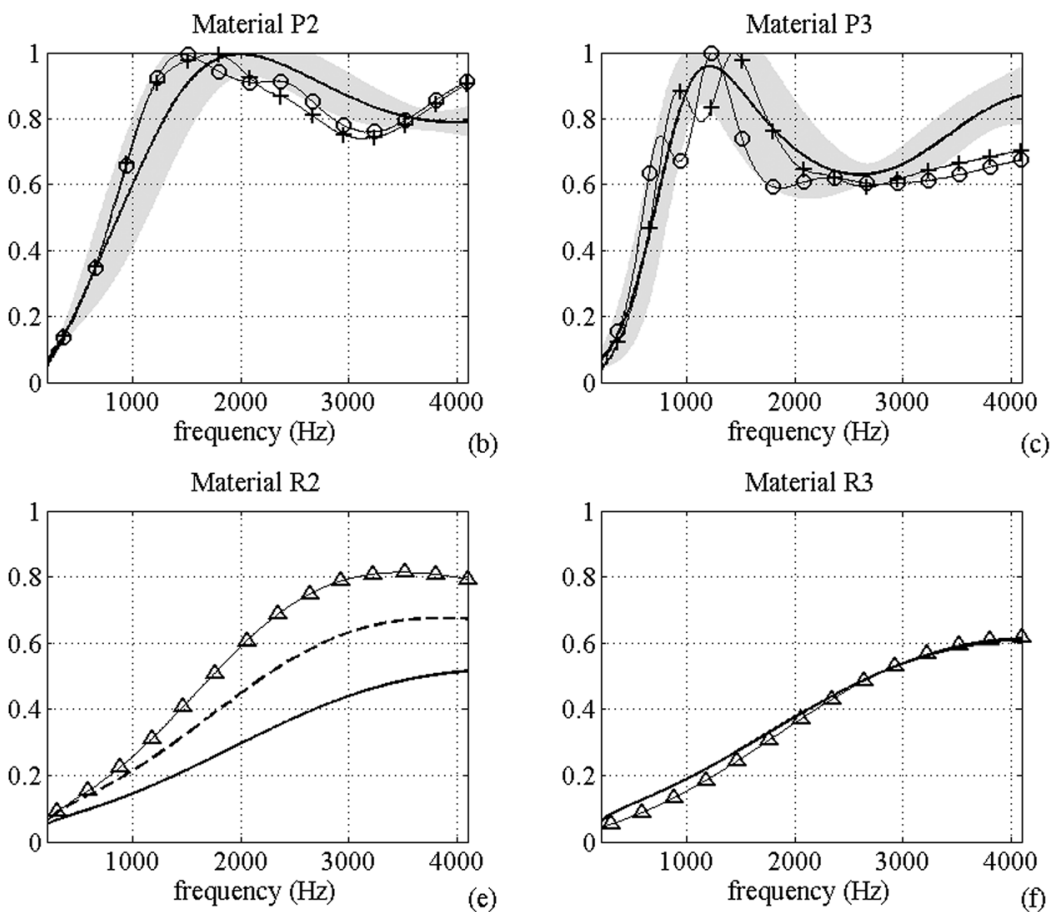

FIG. 6. Sound absorption coefficient of 1 in. thick PU foam layer. Simulations are based on the non-acoustic properties determined from the 2-parameter model: (thick continuous line) JCA model; (grey area) propagation of non-acoustic measurements uncertainties; $(\mathrm{O},+)$ impedance tube measurements; $\Delta$ JCA simulation associated to the measured non-acoustic parameters; (thick discontinuous line) JCA model associated to the 2-parameter model using an equivalent reticulation rate determined from tortuosity measurement (by inverting Eq. (9)). 
gives a mean scale factor $B$ equal to 3.17 which is much coherent with the value found for our foams.

By comparing Figs. 5(a) and 6(a), one can note that making use of the 2-parameter model in place of the 3-parameter model for fully reticulated PU foams decreases significantly the uncertainties related to the estimation of the sound absorption coefficient. This again reveals that the influence of strut dimensions dispersion (associated to the 3-parameter model) is greater compared to the one associated to the two geometric ratios $A$ and $B$; ratios which however account for the microstructure variability between all the foams constituting the first characterization set (i.e., M1-M15 with $21.5 \mathrm{~kg} \cdot \mathrm{m}^{-3}<\rho_{1}<29.5 \mathrm{~kg} \cdot \mathrm{m}^{-3}$ and $\left.96.8 \%<\phi<99 \%\right)$. This effect is less pronounced in the case of partially reticulated foams, meaning that the estimated dispersion on the sound absorption simulations is mainly governed by the dispersion measured on the reticulation rate.

Finally, both the 3-parameter and the 2-parameter models seem unable to correctly predict the acoustic behaviors of foams R1 and R2 (compare triangles with solid line in Figs. 5(d), 5(e), 6(d), and 6(e)). Indeed, these semiphenomenological models do not account for anisotropy effect (foam R2) and the reticulation rate $R_{w}$ of these foams was unknown and set to $100 \%$. However, the presence of membranes for foams R1 and R2 is revealed by a tortuosity measured, respectively, at 1.12 and 1.13 in Ref. 11; which is higher than the mean value of 1.05 found for fully reticulated PU foams in Ref. 12 (see also Sec. III D). An equivalent reticulation rate $R_{w}{ }^{e q}$ can thus be estimated from Eq. (9) since it only depends on the reticulation rate. Note that an anisotropy effect is not expected to provide a tortuosity increase. $R_{w}{ }^{e q}$ is thus found equal to $84 \%$ and $82 \%$ for foams R1 and R2, respectively. This value seems in good agreement with the microstructure pictures given in Ref. 11, especially for foam R1 for which few membranes can be seen on the micrograph. This membrane effect thus explains the slightly larger airflow resistivity of foams R1 and R2 compared to fully reticulated foams with similar cell size as shown in Fig. 2(d). Figures 6(d) and 6(e) confirm that the use of the equivalent reticulation rate improves the estimation of the sound absorption coefficient using the 2-parameter model (compare dashed and solid lines). This analysis highlights the importance of measuring the reticulation rate for a complete characterization of the foam microstructure. In addition, it demonstrates that the 2-parameter model is able to correctly predict the sound absorption behavior of PU foams being fully and partially reticulated, isotropic and anisotropic.

\section{CONCLUSION}

This paper presented a simplification and validation of the semi-phenomenological model recently proposed by the authors ${ }^{12}$ to link the microstructure of highly porous PU foams with their sound absorbing behaviour. The initial model estimates the non-acoustic properties involved in the Johnson-Champoux-Allard model (i.e., porosity $\phi$, airflow resistivity $\sigma$, tortuosity $\alpha_{\propto}, \ldots$ ) from cell shape consideration and measurements of three microstructure properties by use of a specific microscope (SEM, stereomicroscope). This 3-parameter model requires measurements of strut length $l$, strut thickness $t$, and the reticulation rate $R_{w}$ characterizing an ideal cell of the material. Sound absorption predictions using this model is assessed using six new polyurethane foams selected near the limits of the assumptions used to derive it. It is shown that great care should be taken when measuring strut dimensions since it could greatly affect the estimated sound absorption behaviour. In consequence, a simplified 2-parameter semiphenomenological model is proposed. This model only requires measurements of cell size $C_{s}$ and reticulation rate $R_{w}$, assuming that the geometric ratio between strut length and thickness is known. This ratio is given in the paper for highly porous PU foams $(97 \%<\phi<99 \%)$ with relatively low bulk density $\left(21.5 \mathrm{~kg} \cdot \mathrm{m}^{-3}<\rho_{1}<29.5 \mathrm{~kg} \cdot \mathrm{m}^{-3}\right)$ investigated in this work. For other foams, it can be estimated using Eq. (4) and porosity measurements. The 2-parameter model provides similar non-acoustic properties compared to the 3-parameter model but greatly simplifies the microstructure characterization. Furthermore, it correctly estimates the sound absorption behavior of both fully and partially reticulated foams, isotropic and anisotropic. Still, a more complete sensitivity analysis should be performed for both semi-phenomenological models, in order to investigate the correlation between the input parameters and identify the parameters contributing the most to output variability, thus requiring precise measurement. In addition, the applicability of the 2-parameter model on anisotropic foams should also be validated on additional polyurethane materials.

\section{APPENDIX A: POROUS MATERIAL MODELING}

Assuming that the acoustic wavelength is much larger than the characteristic dimensions of the foam (cell size) and that the saturating fluid behaves as an incompressible fluid at the microscopic scale, the air in the porous frame can be replaced by an equivalent fluid. This equivalent fluid is characterized by two complex frequencydependent functions: the dynamic density $\rho(\omega)$ which takes into account the visco-inertial interaction between the frame and the saturating fluid, and the dynamic bulk modulus $K(\omega)$, which takes into account the thermal interaction. In this paper, the homogeneous foam layer is rather described by the following two intrinsic acoustical properties: the wave number $k(\omega)=\omega(\rho(\omega) / K(\omega))^{1 / 2}$ and the characteristic impedance $Z_{c}(\omega)=(\rho(\omega) K(\omega))^{1 / 2}$. For convenience, the explicit dependence on $\omega$ will be omitted, unless necessary. The well-known Johnson ${ }^{19}$ and Champoux-Allard ${ }^{20}$ semi-phenomenological models are used here to predict the frequency behavior of $\rho$ and $K$ since an analytical description of sound propagation that takes into account the complex microstructure geometry of the PU foams is not possible. ${ }^{2}$ According to the JCA model, ${ }^{2,19,20}$ the two dynamic properties are derived from their low to high-frequency asymptotic behaviors using specific viscous and thermal dynamic tortuosity functions, $\alpha(\omega)$ and $\alpha^{\prime}(\omega)$, such as ${ }^{2}$ 


$$
\rho(\omega)=\rho_{0} \alpha(\omega)=\rho_{0}\left(\alpha_{\infty}+\frac{\phi \sigma}{j \omega \rho_{0}}\left[1+\left(\frac{2 \alpha_{\infty} \eta}{\Lambda^{2} \phi \sigma}\right) \frac{j \omega}{v}\right]^{1 / 2}\right)
$$

$$
\begin{aligned}
K(\omega) & =P_{0} /\left(1-\frac{\gamma-1}{\gamma} \alpha^{\prime}(\omega)^{-1}\right) \\
& =P_{0} /\left(1-\frac{\gamma-1}{\gamma}\left(\frac{8 v^{\prime}}{j \omega \Lambda^{\prime 2}}\left[1+\left(\frac{\Lambda^{\prime}}{4}\right)^{2} \frac{j \omega}{v^{\prime}}\right]^{1 / 2}+1\right)^{-1}\right),
\end{aligned}
$$

where $\omega$ is the angular frequency, $\rho_{0}$ is the fluid density, $\eta$ is the fluid viscosity, $\nu=\eta / \rho_{0}=P_{r} \nu^{\prime}, P_{r}$ being the Prandtl number, $P_{O}$ the atmospheric pressure, and $\gamma$ the specific heat ratio. The following non-acoustical parameters are used to describe the visco-thermal and inertial couplings between the porous aggregate and the interstitial fluid on a macroscopic scale: open porosity $\phi$, static air flow resistivity $\sigma$ $\left[\mathrm{N} . \mathrm{s} / \mathrm{m}^{4}\right]$, tortuosity $\alpha_{\propto}$, viscous $\Lambda[\mu \mathrm{m}]$, and thermal characteristic lengths $\Lambda^{\prime}[\mu \mathrm{m}]$.

The normal-incidence sound absorption coefficient of the porous media is derived from the complex reflection coefficient $r$ :

$$
\alpha=1-|r|^{2}, \quad r=\frac{Z_{s}-\rho_{0} c_{0}}{Z_{s}+\rho_{0} c_{0}}
$$

with $c_{O}$ the speed of sound in air and $Z_{S}$ the normal-incidence surface impedance. For a layer of fluid of thickness $d$ and backed by an impervious rigid wall, this impedance is given by $Z_{s}=-j Z_{c} \operatorname{cotg}(k d) / \phi .^{2}$

\section{APPENDIX B: CHARACTERIZATION OF THE NON-ACOUSTIC PROPERTIES}

The porosity $\phi$ and the airflow resistivity $\sigma$ are measured using direct techniques. ${ }^{21,22}$ For both fully and partially reticulated foams, the tortuosity $\alpha_{\propto}$ is estimated from indirect method based on ultrasound techniques. Tortuosity of fully reticulated materials is determined from ultrasonic measurement of transmitted waves. ${ }^{23}$ This latter method being restricted to low resistive materials, the tortuosity $\alpha_{\propto}$ of partially reticulated foams is estimated from the measurement of acoustic waves reflected by a slab of porous material at oblique incidence. ${ }^{24}$ The two characteristic lengths $\left(\Lambda, \Lambda^{\prime}\right)$ and the thermal permeability $k_{0}{ }^{\prime}$ are determined using the indirect characterization method proposed by Panneton and Olny ${ }^{25,26}$ based on the JCA model modified by Lafarge ${ }^{27}$ (also called JCAL). This indirect method requires the measurement of the equivalent dynamic bulk modulus $K_{e q}$ and equivalent dynamic density $\rho_{e q}$ of the tested material performed here using the 3-microphone impedance tube method proposed by Salissou et al. ${ }^{28}$ The Lafarge et al. model $^{27}$ is used in the characterization method to model the equivalent dynamic bulk modulus $K_{e q}$ because it was shown elsewhere to match more closely its low frequency behaviour. ${ }^{2}$ This determination of the parameter $k_{0}^{\prime}$ is mainly used here to lower the error in the determination of the thermal characteristic length $\Lambda^{\prime}$. The thermal permeability parameter $k_{O}{ }^{\prime}$ is discarded from the micro-/macro analysis, at least for the time being, and the classical JCA model is used in the whole paper to simulate the sound absorbing properties of the PU foams.

${ }^{1}$ D. J. Doherty, R. Hurd, and M. G. Walker, "The physical properties of rigid polyurethane foams," Chem. Ind. (London) 30(7), 1340-1356 (1962).

${ }^{2}$ J. F. Allard and N. Atalla, Propagation of Sound in Porous Media: Modeling Sound Absorbing Materials, 2nd ed. (Willey, New York, 2009).

${ }^{3}$ A. Cummings and S. P. Beadle, "Acoustic properties of reticulated plastic foams,” J. Sound Vib. 175(1), 115-133 (1993).

${ }^{4}$ R. F. Lambert, "The acoustical structure of highly porous open-cell foams," J. Acoust. Soc. Am. 72(3), 879-887 (1982).

${ }^{5} \mathrm{P}$. Göransson, "Acoustic and vibrational damping in porous solids," Philos. Trans. Roy. Soc. A 364, 89 (2006).

${ }^{6}$ E. Lind-Nordgren and P. Göransson, "Optimising open porous foam for acoustical and vibrational performance,” J. Sound Vib. 329, 753 (2010).

${ }^{7}$ C. Perrot, R. Panneton, and X. Olny, "Periodic unit cell reconstruction of porous media: Application to an open cell aluminum foam," J. Appl. Phys. 101, 113538 (2007).

${ }^{8}$ C. Perrot, R. Panneton, and X. Olny, "Computation of the dynamic thermal dissipation properties of porous media by Brownian motion simulation: Application to an open-cell aluminum foam," J. Appl. Phys. 102, 074917 (2007).

${ }^{9}$ C. Perrot, F. Chevillotte, and R. Panneton, "Dynamic viscous permeability of open-cell aluminum foam: computation versus experiments," J. Appl. Phys. 103, 024909 (2008).

${ }^{10}$ C. Perrot, F. Chevillotte, and R. Panneton, "Bottom-up approach for microstructure optimization of sound absorbing materials," J. Acoust. Soc. Am. 124(2), 940 (2008).

${ }^{11}$ C. Perrot, F. Chevillotte, M.-T. Hoang, G. Bonnet, F.-X. Bécot, L. Gautron, and A. Duval, "Microstructure, transport, and acoustic properties of opencell foam samples: Experiments and three-dimensional numerical simulations," J. Appl. Phys. 111, 014911 (2012).

${ }^{12}$ O. Doutres, N. Atalla, and K. Dong, "Effect of the microstructure closed pore content on the acoustic behavior of polyurethane foams," J. Appl. Phys. 110, 064901 (2011).

${ }^{13}$ Y. Wang and A. M. Cuitino, "Three-dimensional nonlinear open-cell foams with large deformations," J. Mech. Phys. Solids 48, 961-988 (2000).

${ }^{14}$ Joint Committee for Guides in Metrology, Evaluation of Measurement Data-Guide to the Expression of Uncertainty in Measurement (JCGM 100:2008, Bur. Intl. Poids et Mesures, Sèvres, 2008).

${ }^{15}$ I. P. Dunn and W. A. Davern, "Calculation of acoustic impedance of multi-layer absorbers,” Appl. Acoust. 19(5), 321-333 (1986).

${ }^{16}$ J.-P. Bonnet, F. Topin, and L. Tadrist, "Flow laws in metal foams: Compressibility and pore size effects," Transp. Porous Med. 73, 233-254 (2008).

${ }^{17}$ Anonymous, "Acoustics - Determination of sound absorption coefficient and impedance in impedance tubes. Part 2: Transfer-function method," International Standard ISO-10534-2 (1998).

${ }^{18}$ W.-Y. Jang, A. M. Kraynik, and S. Kyriakides, "On the microstructure of open-cell foams and its effect on elastic properties," Int. J. Solids Struct. 45, 1845-1875 (2008).

${ }^{19}$ D. L. Johnson, J. Koplik, and R. Dashen, "Theory of dynamic permeability and tortuosity in fluid-saturated porous media," J. Fluid Mech. 176, 379 (1987).

${ }^{20}$ Y. Champoux and J.-F. Allard, "Dynamic tortuosity and bulk modulus in air-saturated porous media,” J. Appl. Phys. 70(4), 1975-1979 (1991).

${ }^{21}$ Y. Salissou and R. Panneton, "Pressure/mass method to measure open porosity of porous solids," J. Appl. Phys. 101, 124913.1 (2007).

${ }^{22}$ M. R. Stinson and G. A. Daigle, "Electronic system for the measurement of flow resistance," J. Acoust. Soc. Am. 83, 2422 (1988).

${ }^{23} \mathrm{M}$. Melon and B. Castagnede, "Correlation between tortuosity and transmission coefficient of porous media at high frequency," J. Acoust. Soc. Am. 98, 1228 (1995).

${ }^{24}$ Z. E. A. Fellah, S. Berger, W. Lauriks, C. Depollier, C. Aristégui, and J.-Y. Chapelon, "Measuring the porosity and the tortuosity of porous materials via reflected waves at oblique incidence," J. Acoust. Soc. Am. 113(5), 2424 (2003). 
${ }^{25} \mathrm{R}$. Panneton and X. Olny, "Acoustical determination of the parameters governing viscous dissipation in porous media," J. Acoust. Soc. Am. 119(4), 2027 (2006).

${ }^{26} \mathrm{X}$. Olny and R. Panneton, "Acoustical determination of the parameters governing thermal dissipation in porous media," J. Acoust. Soc. Am. 123(2), 814 (2008).
${ }^{27}$ D. Lafarge, P. Lemarinier, J.-F. Allard, and V. Tarnow, "Dynamic compressibility of air in porous structures at audible frequencies," J. Acoust. Soc. Am. 102, 1995 (1997).

${ }^{28}$ Y. Salissou, R. Panneton, and O. Doutres, "Complement to standard method for measuring normal incidence sound transmission loss with three microphones," J. Acoust. Soc. Am. 131(3), EL 216 (2012). 
Journal of Applied Physics is copyrighted by the American Institute of Physics (AIP). Redistribution of journal material is subject to the AIP online journal license and/or AIP copyright. For more information, see http://ojps.aip.org/japo/japcr/jsp 